\title{
Pseudomembranous Plaque Stomatitis
}

\section{Sody Naimer*}

Department of Family Medicine, Ben-Gurion University of the Negev, Beer-Sheva, Israel

"Corresponding author: Dr. Sody Naimer, Department of Family Medicine, Ben-Gurion University of the Negev, POB 653, Beer-Sheva 84105, Israel, Tel: 972-8-6477433, Fax: 972-8-6477623; E-mail: sodyna@clalit.org.il

\begin{abstract}
Objectives: The oral mucosa is a susceptible component of our anatomy with explicit implications on our well being. Consequently an unfamiliar pattern of oral mucosa pathology will raise much concern. Confronting unrecognized lesions undoubtedly challenges our professional acumen and clinical expertise. In attempt to discern the gravity of an exceptional condition the caring dermatologist may contemplate to indulge any of a number of approaches with different levels of intervention. This paper describes the rationale for choosing a conservative clinical management approach to two children exhibiting unique oral lesions.

Methods: Two youngsters arriving for consultation, presented approximately twelve months apart with similar oral lesions. They were examined and thorough case histories were taken. The findings were photographed, a bacterial culture was taken and follow up ensued.

Results: Both youngsters displayed pseudomembranous plaques covering a limited zone of the oral mucosa. One case involved the inner buccal wall and the next the inferior vestibule. Histories failed to disclose environmental, local, infectious or inflammatory inciting factors contributing to their appearance. Without intervention the lesions resolved spontaneously without further sequelae. The discussion digresses upon the differential diagnosis to be considered setting an outline for a systematic approach to oral mucosal pathology.

Conclusions: This condition's unique characteristics justify its recognition as a novel entity. This report should arouse awareness of the justification of a conservative approach to such cases, whose future investigations and clinical histories may assist to elucidate the etiology standing behind its development.
\end{abstract}

Received date: July 10, 2016

Accepted date: Sept 23, 2016

Published date: Sept 30, 2016

Citation: Naimer, S. Pseudomembranous Plaque Stomatitis. (2016) Invest Dermatol Venerol Res 2(2): 79- 83 .

DOI: $10.15436 / 2381-0858.16 .1010$

Keywords: Mouth mucosa; Stomatitis; Oral cavity; Spontaneous remission

\section{What is already known on this topic:}

1. Oral cavity lesions are extremely diverse and result in pathology which can be classified by historical, morphological, or histopathological characteristics.

2. Clinicians will need to collect all possible information through interview, physical examination and sometimes ancillary testing in order to differentiate between the various mucosal lesions.

3. Much is to be gained if the treating dermatologist can rapidly recognize the presented lesions and allay fears and unnecessary anxiety.

What this paper adds:

1. A systematic morphological approach can prove rewarding and assists in focusing on the precise diagnosis.

2. Conservative management is often justified and often as in these cases the preferable choice.

3. A novel unrecognized condition in need of further investigation demanding predominantly reassurance until spontaneous remission eventually transpires.

\section{Introduction}

Pathological changes of the oral mucosa leading to well defined diagnoses are diverse and usually readily recognized by clinicians of overlapping fields: dermatologists, pediatricians, family physicians as well as ear nose and throat specialists and 
dentists. Accurate diagnosis of oral mucosa lesions is necessary for instituting appropriate management of patients. Lesions of the oral cavity can vary diversely from localized, relatively mild, life quality perturbing, damage to the oral mucosa ${ }^{[1]}$, all the way to rampaging jeopardizing advanced florrid mucositis ${ }^{[2,3]}$.

Despite the current nature of practice in an era characterized by wide adoption of sophisticated technological techniques directed all the way down to the level of the biological micro component, there is absolutely no substitute for the solid basics of medical history and physical examination ${ }^{[1]}$. In the process of formulating a differential diagnosis, the cognitive process of applying logic and knowledge is undertaken in a series of stepby-step analyses, to create a list of possible etiologies. Precautions should be taken that serious conditions are not overlooked. Patients must be escorted throughout the process and should be provided emotional and medical support until full recovery of epithelial integrity occurs, if possible. In this paper, our patients presented stomatitis of a limited pseudomembranous plaque, an unfamiliar pattern, which raised much concern. Pseudomembranous inflammation is a form of exudative inflammation that involves mucous and serous membranes; consisting of relatively large quantities of fibrin in the exudate and resulting in a rather tenacious membrane-like covering that is fairly adherent to the underlying acutely inflamed tissue; the pseudomembrane usually contains (in addition to the dense network of fibrin) varying quantities of plasma protein, degenerated and necrotic elements from the affected tissue, polymorphonuclear leukocytes, bacteria, etc.

In attempt to discern the gravity of the presenting condition the clinician contemplated indulging a conservative rather than a number of other approaches with different levels of intervention. This demonstrates that one must responsibly weigh the benefits against the risks before embarking on the chosen attitude of management.

\section{Material and Methods}

The case histories of two children presenting with irregular oral lesions are detailed. Each referred to the same primary pediatric care service approximately a year apart. Their parents consented to deliver full clinical details and have the lesions photographed for documentation and follow up purposes. A wide differential diagnosis is raised with discussion regarding the most appropriate pathogenesis. The possible pathophysiological conditions entertained relating to these presentations are described and the inferences leading us to believe that this is a separate novel entity is shared. The children's' parents provided full consent to report and publish their images and case details.

\section{Case Details}

Case I: A four year old boy was brought in by his grandfather complaining of 'a pale stain on the inner left cheek'. Upon questioning he attested to discomfort upon contact with his tongue or food. It was not noticed previously and most probably developed as his parents were away on vacation for a number of days without any known inciting factor. Upon examination a uniform, well demarcated lesion was visible along the inner mucosa of the left lower cheek at the level of the dental line (Figure 1). The single lesion close to a rectangular shape $20 \times 7 \mathrm{~mm}$ area extended from the border of the lip posterior into the oral cavity. This solitary plaque maintained the appearance of a cream/ white color shade with membranous texture seemingly superficial enough to be easily brushed away. However, contact with a tongue depressor or culture swab could not separate secretion or exudate from the underlying surface, nor did this result in bleeding of the tissue as commonly seen with macerated lesions. The complete surface of the buccal mucosa was free of any inflammatory or other processes. There was no associated fever and no rash or further involvement of other body systems. Lymphadenopathy could not be detected. The child was previously completely healthy. The family cannot recall any previous incidents and refute any possible traumatic event. There was no exposure to especially hot beverage or food. The parents were requested to report any deterioration, spread or aggravation of symptoms. Culture for microorganisms was not obtained in this case. Symptomatic topical anesthesia was offered with guidance to refrain from irritating food material such as acidic substances in order not to exacerbate the pain. Ten days later the lesion completely disappeared without leaving any scarring or residual complaints. There was no relapse in the ensuing 24 months.

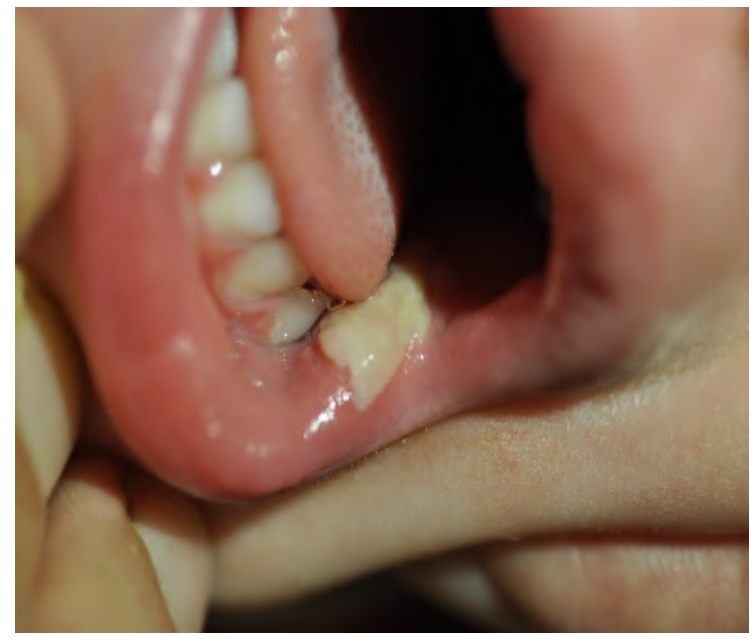

Figure 1: Lesion inner cheek of 3 year old boy.

Case II: A 10- year- old girl arrived with her father complaining of a "bizarre mass" stuck to the inner lower mouth. The lesion occupied the vestibular aspect of the oral mucosa approaching the vermillion border of her lower right lip (Figure 2). Again, history disclosed a completely healthy background without any established trigger leading to the development of the ailment. She claimed not biting her lip and was not exposed to cinnamon or other irritating substance. No associated fever or lymphadenopathy was detected. As previously described, this lesion constituted of a plaque of pseudomembrane that 'sat' on the mucosa like candle wax, so securely that it could not be wiped off. The superficial secretion was not amenable to friction removal with a gauze pad and remained in position without any involvement of additional oral mucosa regions. A bacterial culture of the lesion was unrewarding. The finding seemed not to warrant a fungal culture. After two weeks without intervention the lesion spontaneously disappeared. Following healing no complications were observed. No recurrence has been documented in the following year. 


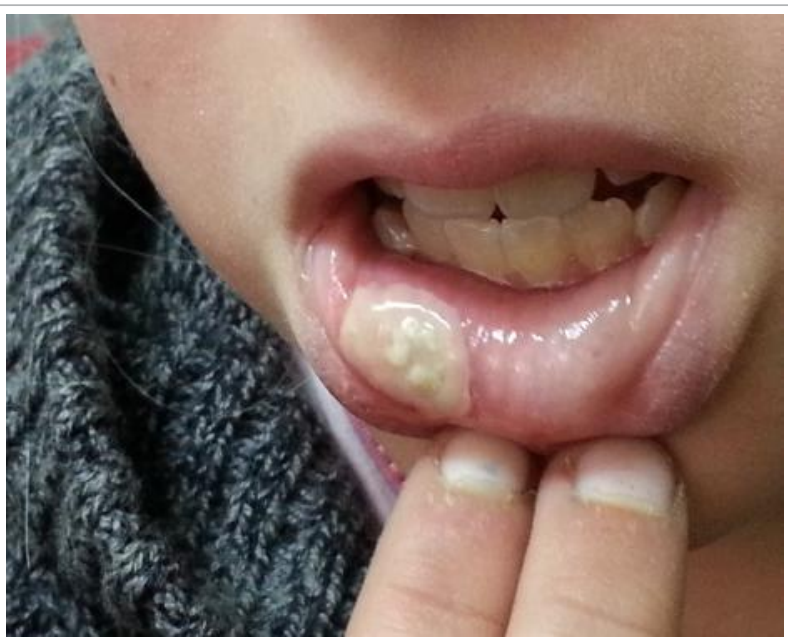

Figure 2: Lesion lower vestibule of 10 year old girl.

\section{Results}

In summary, both youngsters were otherwise healthy and presented with a similar bizarre pseudomembranous plaque covering a limited zone of the oral mucosa. While one case involved the inner buccal wall and the next the inferior vestibule, both reached the external border of the oral orifice. The symptoms were relatively tolerable and consistent with normal behaviour and near regular beverage and nutrition consumption. The conservative approach adopted involved withholding therapy and aside from bacterial cultures refraining from obtaining a tissue diagnosis. This attitude proved rewarding since in both patients the condition resolved spontaneously without any sequelae.

\section{Discussion}

Stomatitis is defined as inflammation of mucous lining of any of the structures in the mouth, cheeks, lip, tongue, gingiva, and floor or roof of the mouth ${ }^{[4]}$. This common phenomenon is often associated with pain, redness, swelling, and sometimes bleeding. Etiologies of such morbidity are multiple and diverse. As our primary concern is to discover neoplastic processes at all costs, the compulsion to achieve tissue diagnosis will be far more pertinent in hyperpigmented lesions of the oral cavity ${ }^{[5,6]}$. Thus, in the vast majority of stomatitis presentations we may rely predominantly upon recognition of lesion appearance and evolution of the clinical course ${ }^{[7]}$.

Knowledge of oral pathology should be organized using a system of classification. This can be based on historical information: duration, triggers, localized and environmental exposures, associated morbidity, previous events and rate of lesion development. As mentioned above, histopathology can contribute much to differentiate between the various lesions; however obtaining this information requires tissue biopsy, an invasive procedure. Much can be learned solely based on a classification dictated by morphological features. Initially, all diagnoses are divided into superficial and deep lesions. If hypertrophy of the tissue is not apparent, we may consider the findings involving either the epithelium or superficial connective tissue of mucosa. Such lesions will not exceed $2-3 \mathrm{~mm}$ in thickness. In our pursuit for the pathogenesis of surface lesions, color intensity is a major characteristic determining pathogenesis. Lesions may be: white, pigmented, or vesicular-ulcerated-erythematous. The brightly colored, appear white, tan, or light yellow. Three subdivisions inside this category are recognized according to the depth of the lesion.

I. Subepithelial: Members of this group include congenital keratotic cysts, scars or Fordyce granules. White lesions due to subepithelial change have normal overlying epithelium, but changes in the connective tissue partially mask blood vessels and cause the area to appear white, yellow or tan. These lesions have a smooth translucent surface, do not rub off, and are not painful ${ }^{[8]}$. In such instances the appearance will not be pseudomembranous under any circumstances. Thus our cases do not fall into the criteria of this group.

II. Epithelial thickening: Lesions of this category appear white because the pink to red color of the blood vessels in the underlying connective tissue is masked by the increased thickness of the epithelium. These lesions are rough to palpation, symptomatic, and gauze will not rub off this layer. They appear flat white when dried. Involvement of the tongue is compulsory in three of these: hairy, hairy leukoplakia, and erythema migrans (geographic) ${ }^{[9]}$. Numerous causes produce pigmentation lesions of oral mucosa. When generalized they are bilateral, multiple and diffuse. In general, the extent of oral mucosal pigmentation is directly related to the extent of pigmentation of the skin. The most common from is hereditary (racial, ethnic, physiologic) pigmentation ${ }^{[10]}$. These forms are chronic.

A localized malignancy such as squamous cell carcinoma or carcinoma in situ is extremely rare at young ages and will continue to erode tissue if left untreated. Evolution is usually slow. Such lesions appear as superficially invasive rather than soft tissue enlargements. Dysplasia, carcinoma in situ, and squamous cell carcinoma can occur on any oral mucosal surface. They are almost invariably non-painful, and thus patients may ignore their existence. Early lesions may be white, rough, epithelial thickening lesions, red persistent non-painful lesions, or a combination of these. Focal keratosis stems from increased thickness of the keratin layer of stratified squamous epithelium with no microscopic evidence of atypical epithelial cells. Clinically, hyperkeratotic lesions appear as white, rough, non-painful patches that do not rub off. They sometimes represent a physiologic response (callus-like) to chronic irritation. They may be secondary to chronic irritation, such as biting. Similarly, epithelial dysplasia appear clinically as white, rough, non-painful areas, or non-painful red patches ("erythroplakia" or "erythroplasia") It is an atypical or abnormal growth of the stratified squamous epithelium lining a mucosal surface. A fraction of lesions diagnosed as epithelial dysplasia will progress to squamous cell carcinoma, while others will resolve ${ }^{[11]}$.

White sponge nevus is an inherited diffusely white, rough, thickened and folded oral mucosa. It is most commonly situated on the buccal mucosa bilaterally, but other oral mucosal areas may be involved. It is not painful. Gray-white, opaque, or milky oral mucosa is probably a variation of normal occurring much more commonly in those with heavily pigmented skin. Leukoedema is a generalized white change of mucosal color rather than a disease. Again, this is asymptomatic and its cause is unknown. Each of the above last from months to years and do not disappear within weeks of presentation. "Leukoplakia" 
refers to a clinically white mucosal thickening most often microscopically shown to be hyperkeratosis. Less than $5 \%$ are associated with malignancy ${ }^{[12]}$. This is not observed in children.

Lichen planus almost always presents with multiple lesions bilaterally, with the buccal mucosa commonly involved. Oral lesions may occur with or without skin lesions and are arranged in a network pattern (Wickham's striae) with erythema of the surrounding mucosa. White patches, erythematous erosions, and ulcers may also occur. The white lesions do not protrude from the tissue surface and are not painful, but the erosions and ulcers are usually painful.

III. Surface debris white lesions: These are associated with necrosis of the overlying epithelium. The necrotic epithelium can be removed with gauze leaving an erythematous or ulcerated base. Surface debris lesions are usually painful. Localized pigmented surface lesions of the oral mucosa will include the most prevalent of oral lesions: candidiasis. This is by far the most common cause of oral discomfort. At this age, it is far more predominant in immunosuppressive states or after antibiotic therapy. These oral lesions manifest in a number of distinct presentations. 'Erythematous' - red macular lesions, often with a burning sensation. 'Perleche' or angular cheilitis- erythematous, scaling fissures at the corner of the mouth. 'Pseudomembranous' - adherent white plaque that are removable. The latter, is the most similar to our scenarios but our children lacked the classic curds that should be able to be scraped off. Likewise, there was not an erythematous or friable mucosa. If left untreated the infection will invariably spread and involve wider surfaces of the oral cavity and gingiva, which was not observed here ${ }^{[13]}$. Finally, fungi will characteristically not exhibit such a distinct clear cut border as was presented in these cases.

Any traumatic event may cause mucosal surfaces to ulcerate and produce a superficial proteinaceous coat of debris. In susceptible individuals these may occur and heal spontaneously as aphthuous ulcers. These are extremely disturbing especially when recurrent. They run a similar time course as we have observed here, however they never appear as uniform, confluent pseudomembranous plaques ${ }^{[14,15]}$. Similar to these lesions, infections: cocksackae's hand-foot-mouth, or primary herpetic infections will present with discrete scattered lesions upon various sites of the mucosal walls. They usually appear round and not elongated. Autoimmune ulceration is recurrent, more widespread and absolutely must exhibit discernible ulceration ${ }^{[16-18]}$. Such mucosal ulceration is equally an essential component in aphthae or drug induced lesions. Burns of oral mucosa can be caused by heat or chemicals. They present as painful ulcers covered by a white to yellow surface. Often the patient can provide a history of a burn to confirm the diagnosis.

Physical factors as nicotinic, cinnamon gum or other allergic and irritant causes ${ }^{[19-22]}$, vitamin deficiencies usually cause atrophy or hypersensitive plaques without typical color change or coating as we consistently found in these youngsters.

These previously healthy individuals exhibited symptoms incompatible with any of the established forms of stomatitis. An extensive literature search discloses only few reports of morphological descriptions that may resemble the presented lesions $^{[23,24]}$. However, the morbidity in those cases was widespread, involving broad surface areas of the oral mucosa, and associated with both systemic symptoms and malnutrition. There- fore, this condition's unique characters justify its recognition as a novel entity.

\section{Conclusions}

Oral mucosal lesions represent many conditions that affect all segments of the pediatric population. While some disorders are pathognomonic, others may have similar features that demand to delve deep into additional clinical parameters such as chronicity, number of lesions, dispersion and recurrence. The unique characters of the cases described here, encompassing the lesions' extent, duration, symptomatology and consistency lead us to the conclusion that we face a novel entity incompatible with any previously recognized pattern.

While relieved by the favorable outcome of such oral lesions, the pathogenesis of this entity remains enigmatic. This initial report should serve to arouse awareness of such cases in the future, whose investigations and clinical histories may assist to elucidate precisely whether an infectious, environmental or behavioral etiology stands behind their development.

\section{Acknowledgments: None}

\section{Conflict of interests: None}

\section{Financial and material support: None}

\section{Prior presentations: None}

\section{References}

[1] Scully, C., Sonis, S., Diz, P.D. Oral mucositis. (2006) Oral Dis 12(3): 229-241.

[2] Belfield, P.M., Dwyer, A.A. Oral complications of childhood cancer and its treatment: current best practice. (2004) Eur J Cancer 40(7): 1035-1041.

[3] Carl, W. Oral complications of local and systemic cancer treatment. (1995) Curr Opin Oncol 7(4): 320-324.

[4] Neville, B.W., Damm, D.D., Allen, C.M., et al. Oral and Maxillofacial Pathology, 3rd ed., W.B. Saunders Co., Philadelphia, 2009.

[5] Kauzman, A., Pavone, M., Blanas, N., et al. Pigmented lesions of the oral cavity: review, differential diagnosis, and case presentations. (2004) J Can Dent Assoc 70(10): 682-683

[6] Meleti, M., Vescovi, P., Mooi, W.J., et al. Pigmented lesions of the oral mucosa and perioral tissues: a flow-chart for the diagnosis and some recommendations for the management. (2008) Oral Surg Oral Med Oral Pathol Oral Radiol Endod 105(5): 606-616.

[7] Tsiros, E.P. On the etiology of pseudo membranous inflammations of the oral cavity and their differential diagnosis. (1960) Hellenike Iatrike 29: 880-888.

[8] Dilley, D.C., Siegel, M.A., Budnick, S. Diagnosing and treating common oral pathologies. (1991) Pediatr Clin North Am 38(5): 12271264.

[9] Reamy, B.V., Derby, R., Bunt, C.W. Common tongue conditions in primary care. (2010) Am Fam Physician 81(5): 627-634.

[10] Eisen, D. Disorders of pigmentation in the oral cavity. (2000) Clin Dermatol 18(5): 579-587.

[11] Regezi, J.A., Sciubba, J.J., Jordon, R.C.K. Oral Pathology: Clinical Pathologic Correlations, 4th ed., Saunders Co., Philadelphia, (2003).

[12] Sapp, J.P., Eversole, L.R., Wysocki, G.P. Contemporary Oral and Maxillofacial Pathology, 2nd ed., Mosby, (2003).

[13] Stoopler, E.T., Sollecito, P. Oral mucosal diseases Evaluation and management. (2014) Med Clin N Am 98 (6): 1323-1325. 
[14] Vincent, S.D., Lilly, G.E. Clinical, historic, and therapeutic features of aphthous stomatitis. Literature review and open clinical trial employing steroids. (1992) Oral Surg Oral Med Oral Pathol 74(1): 7986.

[15] Woo, S., Sonis, S.T. Recurrent aphthous ulcers: A review of diagnosis and treatment (1996) JADA 127(8): 1202-1213.

[16] Darling, M.R., Daley, T. Blistering mucocutaneous diseases of the oral mucosa--a review: part 1. Mucous membrane pemphigoid. (2005) J Can Dent Assoc 71(11): 851-854.

[17] Darling, M.R., Daley, T. Blistering mucocutaneous diseases of the oral mucosa - A review: Part 2. Pemphigus vulgaris. (2006) J Can Dent Assoc 72(1): 63-66.

[18] Vincent, S.D., Lilly, G.E., Baker, K.A. Clinical, historic, and therapeutic features of cicatricial pemphigoid. A literature review and open therapeutic trial with corticosteroids. (1993) Oral Surg Oral Med Oral Pathol 76(4): 453-459.

[19] Georgeakopoulos, E.A. Cinnamon contact stomatitis. (2010) J Dermatol Case Rep 4(2): 28-29.

[20] Torgerson, R.R., Davis, M.D., Bruce, A.J., et al. Contact allergy in oral disease. (2007) JAmAcad Dermatol 57(2): 315-321.

[21] Calapai, G., Miroddi, M., Mannucci, C., et al. Oral adverse reactions due to cinnamon-flavoured chewing gums consumption. (2014) Oral Dis 20(7): 637-643.

[22] Fisher, A.A. Reactions of the mucous membrane to contactants, In: N.L. Novick, eds. (1987) Clinics in Dermatology 5(2): 123-136.

[23] Jackson, J.A. Bismuth pseudo membranous stomatitis: Report of a case, (1966) Oral Surg Oral Med Oral Pathol 21(2): 154-157.

[24] Langille, J.A. Acute membranous stomatitis and conjunctivitis. (1944) Can Med Ass J 50(2): 141-143. 